\title{
NON-POINT TRANSFORMATIONS IN CLASSICAL MECHANICS
}

\author{
R. P. Gaida * \\ Institute for Condensed Matter Physics \\ of the National Academy of Sciences of Ukraine \\ 1 Svientsitskyi Str., Lviv, UA-290011, Ukraine \\ (Received September 28, 1998)
}

\begin{abstract}
The problem of applicability of the non-point transformations in the Lagrangian and Newtonian formalism of classical mechanics is investigated. These transformations correspond to non-point canonical transformations and belong to the class of transformations tangent to a given system of second-order differential equations. The methods for constructing such transformations are considered. Two theorems concerning non-point transformations in Hamilton's variational principle and connection between transformed and initial Lagrangians are proved. The relation of the results to canonical transformations in Hamiltonian formalism is discussed. The general results are illustrated by a simple example.
\end{abstract}

Key words: non-point transformation, tangent transformation, canonical transformation, Lagrangian mechanics.

PACS numbers: 03.30.+p, 45.20.Jj

\section{INTRODUCTION}

In the Lagrangian formalism of analytical mechanics the point transformations of generalized coordinates $q_{i}$ acting in the configuration space of a given system are widely used. At the same time, the class of canonical transformations typical for the Hamiltonian formalism may also include non-point transformations, i.e. transformations of a phase space in which transformed coordinates are expressed not only in terms of initial coordinates but depend also on the initial canonical momenta $p_{i}$. Then two questions arise. Firstly, what nonpoint transformations of Lagrangian variables can be associated with such canonical transformations? Secondly, what is the relation between two Lagrangians corresponding to canonically transformed and untransformed Hamiltonians.

In Newtonian mechanics these questions are of no particular interest. However, in the relativistic theory the situation is fundamentally different. Non-point transformations of spatial particle coordinates arise naturally when constructing the single-time three-dimensional Poincaré-invariant description of a directly interacting particle system [1-4]. The non-point transformations are also inevitable when investigating the separation of the internal motion of a relativistic particle system from the motion of the system as a whole in terms of Lagrangian center-of-mass variables [5]. Thus, this problem demands further investigation. A preliminary communication on this subject was published in Ref. [6]. It should be noted that in a recent paper [7] the conditions have been considered under which it is possible to construct transformations of Lagrangian variables which are equivalent to canonical transformations. That approach also permits to establish the connection between transformed and untransformed Lagrangians. Further work is required along these lines because only a special case of infinitesimal transformations is considered in [7].

The structure of the present paper is as follows. In Section II the problem is formulated starting from the assertion that the need for non-point transformations in the Lagrangian formalism follows from the existence of nonpoint canonical transformations. Section III is devoted to the consideration of various ways of constructing the non-point transformations of the generalized coordinates $q_{i}(t)$ which satisfy a certain set of second-order differential equations. In Sec. IV we assume that these equations are Euler-Lagrange equations of Hamilton's variational principle. Under this assumption, we show that the new generalized coordinates $Q_{i}(t)$ are the extremals of a variational principle with higher (third) order derivatives. In Section $\mathrm{V}$ a theorem is formulated which allows to find the standard (no higher order derivatives) Lagrangian as a function of new variables connected with the old variables by non-point transformations provided the initial Lagrangian is given. This procedure is based on the use of a certain auxiliary function $F(q, Q, t)$. In terms of the Hamiltonian, this function is the generating function of the corresponding canonical transformations. Our general results are illustrated by a simple example. Calculations omitted in the main text are presented in the Appendices.

\footnotetext{
* Editorial Note. This paper by the late Professor Gaida (1928-1998) was available only as Preprint of the Institute for Condensed Matter Physics (ICMP-94-5E, Lviv, 1994). We publish this paper on the basis of the preprint text taking into account some corrections contained in the typed manuscript from the archives of Professor Gaida.
} 


\section{NON-POINT TRANSFORMATION IN LAGRANGIAN MECHANICS AS A COUNTERPART OF CANONICAL TRANSFORMATION}

It is well known that the Lagrange equations of classical mechanics are covariant with respect to arbitrary point transformations of generalized coordinates $q_{i}$. In other words, they are covariant with respect to the transformations of the configuration space $\mathbb{E}=\mathbb{R}^{n}$ :

$$
F: \mathbb{E} \rightarrow \mathbb{E}, \quad q \mapsto Q=F(q)
$$

where $q=\left\{q_{1} \ldots, q_{r}\right\}, Q=\left\{Q_{1} \ldots, Q_{r}\right\}$, and $r$ is the number of the degrees of freedom. (In the case of $N$ particle system $r=3 N$ ). This means that the Lagrange equations for the functions $q_{i}(t)$

$$
\frac{d}{d t} \frac{\partial \mathcal{L}}{\partial \dot{q}_{i}}-\frac{\partial \mathcal{L}}{\partial q_{i}}=0, \quad \mathcal{L}=\mathcal{L}(q, \dot{q}, t)
$$

turn into equations of the same form in terms of new variables $Q_{j}(t)$ :

$$
\frac{d}{d t} \frac{\partial L}{\partial \dot{Q}_{j}}-\frac{\partial L}{\partial Q_{j}}=0, \quad L=L(Q, \dot{Q}, t),
$$

where

$$
Q_{j}=F_{j}(q, t), \quad q_{i}=f_{i}(Q, t)
$$

The connection between the generalized velocities can be obtained from relations (2.4)

$$
\begin{gathered}
\dot{q}_{i}(Q, \dot{Q}, t) \equiv \frac{d}{d t} f_{i}(Q, t)=\frac{\partial f_{i}}{\partial t}+\frac{\partial f_{i}}{\partial Q_{j}} \dot{Q}_{j} \\
\dot{Q}_{i}(q, \dot{q}, t) \equiv \frac{d}{d t} F_{i}(q, t)=\frac{\partial F_{i}}{\partial t}+\frac{\partial F_{i}}{\partial q_{j}} \dot{q}_{j}
\end{gathered}
$$

The repeated indices are summation indices.

It is widely accepted that one of the advantages of the Hamiltonian formalism, in comparison with the Lagrangian, is the possibility of using a wider class of the canonical transformations of phase space

$$
\begin{gathered}
Q_{i}=\Phi_{i}^{*}(q, p, t), \quad P_{i}=\Psi_{i}^{*}(q, p, t), \\
q_{j}=\varphi_{j}^{*}(Q, P, t), \quad p_{j}=\psi_{j}^{*}(Q, P, t),
\end{gathered}
$$

with respect to which canonical Hamilton's equations

$$
\dot{q}_{j}=\frac{\partial \mathcal{H}(q, p, t)}{\partial p_{j}} \quad(a), \quad \dot{p}_{j}=-\frac{\partial \mathcal{H}(q, p, t)}{\partial q_{j}}
$$

remain invariant:

$$
\begin{aligned}
& \dot{Q}_{j}=\frac{\partial H(Q, P, t)}{\partial p_{j}} \quad(a), \\
& \dot{P}_{j}=-\frac{\partial H(Q, P, t)}{\partial q_{j}} \quad(b) .
\end{aligned}
$$

Inasmuch as there exists a standard way of transition from the Hamiltonian formalism to the Lagrangian (and vice versa) on the basis of the Legendre transformation, such a transition can be considered in old as well as in new variables. Hence the problem can be posed about the connection between the corresponding Lagrangians $\mathcal{L}\left(q_{i}, \dot{q}_{i}, t\right)$ and $L\left(Q_{i}, \dot{Q}_{i}, t\right)$ and between old and new Lagrangian variables. It should be noted that the conditions for Hamiltonians and canonical transformations (2.6) and (2.7) allowing such a procedure were investigated recently in [7]. These conditions are:

$$
\left\|\frac{\partial^{2} \mathcal{H}}{\partial p_{i} \partial p_{j}}\right\| \neq 0 \quad(a), \quad\left\|\frac{\partial^{2} H}{\partial P_{i} \partial P_{j}}\right\| \neq 0 \quad(b) .
$$

They express the requirement that the set of equations (2.8a) and (2.9a) can be solved with respect to canonical momenta $p_{i}$ and $P_{j}$, respectively:

$$
p_{i}=\omega_{i(\mathcal{H})}(q, \dot{q}, t), \quad P_{j}=\Omega_{j(H)}(Q, \dot{Q}, t)
$$

Assuming that these conditions are fulfilled we can derive from (2.6) and (2.7) the following set of relations:

$$
\begin{gathered}
Q_{i}=\Phi_{i}(q, \dot{q}, t) \quad(a), \quad \dot{Q}_{i}=\Psi_{i}(q, \dot{q}, t), \quad(b) ; \\
q_{j}=\varphi_{j}(Q, \dot{Q}, t) \quad(a), \quad \dot{q}_{j}=\psi_{j}(Q, \dot{Q}, t) \quad(b) .
\end{gathered}
$$

We call these non-point transformations of Lagrangian variables. It is important to emphasize that to obtain the transformations (2.12) and (2.13) one must use Hamilton's equations (2.8a) and (2.9a). (This fact is denoted in (2.11) by the indices $\mathcal{H}$ and $H$ ). Therefore unlike canonical transformations (2.6) and (2.7) of phase space, the form of the relations (2.12) and (2.13) depends on the expressions for the Hamiltonians $\mathcal{H}$ or $H$, and, consequently, for Lagrangians $\mathcal{L}$ or $L$. Thus, different non-point transformations of Lagrangian variables correspond generally to given canonical transformations of the phase space for different physical systems with the fixed number of degrees of freedom. Using the terminology of differential geometry, we can say that these transformations act in the tangent bundle $T \mathbb{E}$ of the configuration space of a system.

Thus, on the basis of Hamiltonian formalism and under assumption that conditions (2.10) are fulfilled we can 
assert that Lagrange function $L(Q, \dot{Q}, t)$ and proper Lagrange equations $(2.3)$ can be correlated to every Lagrange function $\mathcal{L}(q, \dot{q}, t)$ and to equations (2.2) where variables $\{q, \dot{q}\}$ and $\{Q, \dot{Q}\}$ are connected by relations of the general form (2.12) or (2.13) whose specific form depends generally on the choice of $\mathcal{L}$ or $L$.

The questions arise: how can one construct such a bijective correspondence

$$
\tilde{F}: T \mathbb{E} \rightarrow T \mathbb{E}, \quad\{q, \dot{q}\} \mapsto\{Q, \dot{Q}\}
$$

as well as the connection between Lagrangians $\mathcal{L}(q, \dot{q}, t)$ and $L(Q, \dot{Q}, t)$ without using canonical formalism? Inasmuch as in these relations the form of $\mathcal{L}$ (or $L$ ) and, correspondingly, the form of Lagrange equations for functions $q_{j}(t)$ (or $Q_{i}(t)$ ), is reflected, the problem may be formulated as follows.

Let $\mathfrak{M}$ be the set of the functions $t \mapsto q(t)=$ $\left\{q_{1}(t), \ldots, q_{r}(t)\right\}, t \in \mathbb{R}$, which are solutions of the given set of second-order differential equations of motions that can be considered as the set of Euler-Lagrange equations for the variational principle with Lagrangian $\mathcal{L}$ (the set of equations of motion in mechanics). Let, further, $t \mapsto Q(t)=\left\{Q_{1}(t), \ldots, Q_{r}(t)\right\}, t \in \mathbb{R}$, be new functions introduced by means of relations $(2.12 \mathrm{a})$ or $(2.13 \mathrm{a})$. The task is as follows:

$1^{\circ}$. To construct the map (2.14) and the equations for the functions $Q_{i}(t)$;

$2^{\circ}$. To find the Lagrangian $L(Q, \dot{Q}, t)$ for which the Euler-Lagrange equations coincide with the equations for $Q_{i}(t)$.

As it had been noted in the Introduction, the nonpoint transformations of the form (2.12) and (2.13) arise inevitably in the relativistic direct interaction theory in the framework of single-time three-dimensional Lagrangian and Newtonian formalisms [1-4]. This is a consequence of the noninvariance of the notion of the simultaneity of spatially separated events with respect to the Poincaré group, on the one hand, and of using this notion in all Lorentz reference frames, on the other. In such an approach to the construction of the relativistic mechanics of an interacting particle system, transition from the "old" simultaneity to the "new" one is needed. When considering an infinitesimal Lorentz transformation, this transition is performed by means of an infinitesimal shift along particle world lines, which gives rise to derivatives $\dot{x}_{a}^{i}$ in the formulae for transformations of particle coordinates $x_{a}^{i}(a=1, \ldots, N ; i=1,2,3)$ [1-4]. This fact, as well as the problem of the construction of the Lagrangian relativistic center-of-mass variables [5], stimulated the author to investigate the problem of the non-point transformations in classical mechanics.

\section{NON-POINT TRANSFORMATIONS IN DIFFERENTIAL EQUATIONS}

Let functions $q_{i}(t)(i=1, \ldots, r)$ satisfy the set of $r$ second-order differential equations:

$$
f_{k}(q, \dot{q}, \ddot{q}, t)=0, \quad k=1, \ldots, r
$$

Supposing that the determinant $\operatorname{det}\left\|\partial f_{k} / \partial q_{i}\right\| \neq 0$, we may deal with the set of equations

$$
\ddot{q}_{j}-\mu_{j}(q, \dot{q}, t)=0, \quad j=1, \ldots, r
$$

instead of (3.1). We consider the first part of the problem formulated in Section II (i.e. the problem $1^{\circ}$ ) in two cases:

(A) the relations $(2.12 \mathrm{a})$ are given;

(B) the relations (2.13a) are given.

In case (A) the solution of the problem is obtained easily. Differentiating $(2.12 \mathrm{a})$ by $t$ we find

$$
\dot{Q}_{j} \equiv \frac{d \Phi_{j}(q, \dot{q}, t)}{d t}=Z_{j}(q, \dot{q}, \ddot{q}, t)
$$

Using here (3.2) we come to (2.12b). In order to find the relations $(2.13)$ which are inverse to $(2.12)$ we suppose that in the arbitrary point $(t, q, \dot{q}) \in \mathbb{R} \times T \mathbb{E}$ the condition

$$
\left|\begin{array}{cccccc}
\frac{\partial \Phi_{1}}{\partial q_{1}} & \ldots & \frac{\partial \Phi_{1}}{\partial q_{r}} & \frac{\partial \Phi_{1}}{\partial \dot{q}_{1}} & \ldots & \frac{\partial \Phi_{1}}{\partial \dot{q}_{r}} \\
\vdots & & \vdots & \vdots & & \vdots \\
\frac{\partial \Phi_{r}}{\partial q_{1}} & \ldots & \frac{\partial \Phi_{r}}{\partial q_{r}} & \frac{\partial \Phi_{r}}{\partial \dot{q}_{1}} & \ldots & \frac{\partial \Phi_{r}}{\partial \dot{q}_{r}} \\
\frac{\partial \Psi_{1}}{\partial q_{1}} & \ldots & \frac{\partial \Psi_{1}}{\partial q_{r}} & \frac{\partial \Psi_{1}}{\partial \dot{q}_{1}} & \ldots & \frac{\partial \Psi_{1}}{\partial \dot{q}_{r}} \\
\vdots & & \vdots & \vdots & & \vdots \\
\frac{\partial \Psi_{r}}{\partial q_{1}} & \ldots & \frac{\partial \Psi_{r}}{\partial q_{r}} & \frac{\partial \dot{\Psi}_{r}}{\partial \dot{q}_{1}} & \ldots & \frac{\partial \dot{\Psi}_{r}}{\partial \dot{q}_{r}}
\end{array}\right| \neq 0
$$

for the Jacobian of the transformations (2.12) is fulfilled. Then, solving the set of equations (2.12) with respect to $q_{i}, \dot{q}_{i}$ we obtain the formulae $(2.13)$.

The differential equations for $Q_{j}(t)$ can be obtained in two equivalent ways. The first one consists in differentiating equations (2.13a) by $t$ followed by equating the right-hand parts to the corresponding expressions in the right-hand parts of relations $(2.13 \mathrm{~b})$. The second way consists in the differentiation of equalities (2.12b) by $t$, followed by using the equations (3.2) and (2.13) in the right-hand parts.

Problem (B) is more complicated: in this case the relations

$$
\dot{q}_{i} \equiv \frac{d \varphi_{i}(Q, \dot{Q}, t)}{d t}=\zeta_{i}(Q, \dot{Q}, \ddot{Q}, t)
$$

cannot be derived directly to form (2.13b) because second-order differential equations for $Q(t)$ allowing to express $Q_{j}(t)$ in terms of $\{Q, \dot{Q}\}$, are unknown. Differentiating (3.5) by $t$ once more and using (3.2) in the 
left-hand part, and then (2.13a) and (3.5), we obtain a certain set of third-order equations for $Q_{j}(t)$,

$$
\tilde{F}_{k}(Q, \dot{Q}, \ddot{Q}, \dddot{Q}, t)=0, \quad k=1, \ldots, r
$$

Inasmuch as the latter equations are the consequence of Eqs. (3.2) and (2.13a), each solution $\left\{Q_{1}(t), \ldots, Q_{r}(t)\right\}$ of Eqs. (3.6) may be regarded as a counterpart of the initial functions $\left\{q_{1}(t), \ldots, q_{r}(t)\right\}$ corresponding to nonpoint transformations of the form (2.14), one half of which is given by (2.13a) and the second half is to be found. To solve the latter task we can try to construct a certain set of second-order equations

$$
F_{k}(Q, \dot{Q}, \ddot{Q}, t)=0
$$

which have solutions $\left\{Q_{1}(t), \ldots, Q_{r}(t)\right\}$ satisfying also Eqs. (3.6). Supposing that the set of equations (3.7) can be solved with respect to variables $Q_{i}$, we can write Eqs. (3.8) in the form of

$$
\ddot{Q}_{i}-\mathcal{M}_{i}(Q, \dot{Q}, t)=0, \quad k=1, \ldots, r
$$

and reduce problem (B) to problem (A).

Now the question arises in what way equations (3.7) can be constructed. To begin with we notice that general solution of Eqs. (3.6) contains $3 r$ arbitrary constants of integrations, whereas such a solution of Eqs. (3.7) is determined by $2 r$ constants. Therefore to exhaust all possible solutions of Eqs. (3.6) a certain class of Eqs. (3.7) which can be parametrized by $r$ arbitrary constants should be considered.

We consider now some ways of constructing equations (3.7). One of the possibilities consists in finding $r$ first integrals of set (3.6). Corresponding equations of the type (3.7) will contain $r$ arbitrary constants $C_{i}(i=1, \ldots, r)$. Each choice of numerical values of the constants determines a certain map (2.14). If these constants are not fixed they will enter relations (2.12) and (2.13) as parameters, and we obtain $r$-parametric class of non-point transformations.

We can come to an analogous conclusion on the basis of another reason. If the functions $q_{i}(t)$ are considered to be known then relations (2.13a) can be regarded as the set of the first-order differential equations for unknown functions $Q_{j}(t)$. The set of the solutions of this set contains $r$ arbitrary constants $C_{i}$.

Let us note one more way to solve problem (B). Let given functions $\varphi_{i}(Q, \dot{Q}, t)$ define the relations (2.13a) and functions $\Phi_{j}(q, \dot{q}, t)$ to be sought define unknown inverse equalities (2.12a). Then, taking into account (3.2) we have:

$$
\dot{Q}_{i}=\frac{\partial \Phi_{i}}{\partial q_{k}} \dot{q}_{k}+\frac{\partial \Phi_{i}}{\partial \dot{q}_{k}} \mu_{k}(q, \dot{q}, t)+\frac{\partial \Phi_{i}}{\partial t}
$$

Inserting (3.8) into (2.13a) we obtain

$$
q_{j}=\varphi_{j}\left(\Phi(q, \dot{q}, t), \frac{\partial \Phi}{\partial q_{k}} \dot{q}_{k}+\frac{\partial \Phi}{\partial \dot{q}_{k}} \mu_{k}(q, \dot{q}, t)+\frac{\partial \Phi}{\partial t}, t\right)
$$

The equalities (3.10) form a set of first-order partial differential equations which may be used as a basis for finding the functions $\Phi_{j}(q, \dot{q}, t)$.

It should be noticed that problem $(B)$ can be reduced to problem (A) in the important (from the physical point of view) case of such infinitesimal transformations which are non-point transformations owing only to small terms. Indeed, let equations (3.2) be given and let formulae (2.13a) have the form

$$
q_{i}=\varphi_{i}^{(0)}(Q, t)+\epsilon_{i}^{k} \varphi_{k}^{(1)}(Q, \dot{Q}, t)
$$

where $\epsilon_{i}^{k}(i, k=1, \ldots, r)$ are small parameters. Then the arguments of the functions $\varphi_{k}^{(1)}$ can be expressed through $q$ and $\dot{q}$ by the equalities

$$
Q_{j} \approx \Phi_{j}^{(0)}(q, t), \quad \dot{Q}_{j} \approx \frac{d \Phi_{j}^{(0)}}{d t} \equiv \xi^{(0)}(q, \dot{q}, t)
$$

in which $\Phi_{j}^{(0)}(q, t)$ define point transformations that are inverse to the zero-approximation in (3.11). Solving the algebraic set of equations, obtained in this way from (3.11), with respect to $Q_{j}$ we find in the first approximation the equalities of the type (2.12a):

$$
Q_{j} \approx \Phi_{j}^{(0)}(q, t)+\epsilon_{i}^{k} \Phi_{k}^{(1)}(q, \dot{q}, t)
$$

We will illustrate the above by a simple example. Let function $x(t)$ satisfy the equation of motion

$$
\ddot{x}+x=0
$$

and let problem (A) be formulated by setting the transformation

$$
X=\frac{1}{2}(x-\dot{x})
$$

Taking into account equation (3.13), we obtain:

$$
\dot{X}=\frac{1}{2}(x+\dot{x})
$$

From the set of equations (3.14) and (3.15) we find the inverse transformations

$$
x=X+\dot{X} \quad(a), \quad \dot{x}=\dot{X}-X \quad(b) .
$$

Differentiating equality (3.15) by $t$ and using (3.13) and (3.16) we obtain the equation for the function $X(t)$ : 


$$
\ddot{X}+X=0 .
$$

We will consider now problem (B), i.e. we set transformation (3.16a) for the functions $x(t)$ which satisfy equation (3.13). Three methods described above will be used.

1. Using relation (3.16a) in (3.13) we obtain the thirdorder equation for function $X$ :

$$
\dddot{X}+\ddot{X}+\dot{X}+X=0 \text {. }
$$

It is easy to see that each solution of the second-order equation

$$
\ddot{X}+X-2 C e^{-t}=0,
$$

where $C$ is an arbitrary constant (the first integral of Eq. (3.18)), satisfies also Eq. (3.18). Therefore, Eq. (3.19) may be used now to construct the sought map $(x, \dot{x}) \mapsto$ $(X, \dot{X})$ in the same way as in problem (A). Differentiating (3.16a) and using (3.19) we find:

$$
\dot{x}=\dot{X}-X+2 C e^{-t} .
$$

The transformations which are inverse to (3.16a) and (3.20) have the form:

$$
\begin{aligned}
& X=\frac{1}{2}(x-\dot{x})+C e^{-t} \quad(a), \\
& \dot{X}=\frac{1}{2}(x+\dot{x})-C e^{-t} \quad(b) .
\end{aligned}
$$

Thus, the solution of problem (B) leads in our example to one-parametric class of transformations (3.16a), (3.20), and (3.21). Specifically, choosing $C=0$ we return to the relations $(3.14)-(3.16)$.

2. Let us consider relation (3.16a) as the differential equation for the function $X(t)$ with a given function $x(t)$. Integrating this equation by the method of variation of constant we find the relation

$$
X=e^{-t} \int x(t) e^{t} d t+C e^{-t}
$$

where $C$ is an integration constant. Double integration in equality (3.22) by parts and use of the equation (3.13) leads to relations $(3.21)$.

3. Looking for the variable $X$ in the form $X=$ $\Phi(x, \dot{x}, t)$ and taking into account $(3.13)$ we can transform (3.16a) as follows:

$$
\Phi+\dot{x} \frac{\partial \Phi}{\partial x}-x \frac{\partial \Phi}{\partial \dot{x}}+\frac{\partial \Phi}{\partial t}=x
$$

General solution of the latter equation has the form

$$
\Phi=-\frac{1}{2}(\dot{x}-x)+\Phi_{0}(\mathcal{E}, z-t) e^{-z}
$$

where $\Phi_{0}$ is an arbitrary function of two arguments,

$$
\mathcal{E}=x^{2}+\dot{x}^{2}, \quad z=\arcsin (x / \sqrt{\mathcal{E}}) .
$$

Owing to equation $(3.13) \mathcal{E}$ is a constant, $d z / d t=1$, and $z-t=$ const. Therefore, $\Phi_{0}(\mathcal{E}, z-t) e^{-z}=e^{-t}$ const and Eq. (3.24) coincides with (3.21a).

We will make two terminological remarks.

Remark 1. We have seen that the set of equations (3.7) is the consequence of the sets (3.2) and (2.12a) or (2.13a) and, vice versa, the set (3.2) may be considered as the consequence of the sets (3.7) and (2.12a) or (2.13a). Then, accordingly to the definition formulated in [8] each of the sets of first-order equations (2.12a) and (2.13a) is a Bäcklund transformation connecting the sets (3.2) and (3.7).

Remark 2. Let $t \in \mathbb{R}, q=\left\{q_{i}\right\}, v=\left\{v_{i}\right\}, w=\left\{w_{i}\right\}$, $(i=1, \ldots, r)$ be the coordinates of a point of the $3 r-$ dimensional space $\mathbb{E}$, and let $K$ be the manifold in $\mathbb{R} \times \mathbb{E}$ which is defined by the set of equations:

$$
w_{j}-\mu_{j}(q, v, t)=0, \quad j=1, \ldots, r
$$

The transformations in $\mathbb{R} \times \mathbb{E}$

$$
\Xi:(q, v, w, t) \mapsto(Q, V, W, t)
$$

are called tangent transformations with respect to the manifold $K$ if they maintain on $K$ the relations:

$$
d q_{i}-v_{i} d t=0, \quad d v_{i}-w_{i} d t=0 \quad(i=1, \ldots, r)
$$

We call the transformations

$$
Q_{i}=\Phi_{i}(q, v, t), \quad V_{i}=\Psi_{i}(q, v, t)
$$

tangent transformation with respect to the set of equations (3.2) if they are induced in $K$ by transformations (3.27), which are tangent transformations with respect to manifold $K[9,10]$. Thus we can say that relations (2.12) and (2.13), which are constructed in one of the ways considered above, define the tangent transformations with respect to the set of equations (3.2). In accordance with the results of [11] they cannot be tangent transformations in the whole space $\mathbb{R} \times \mathbb{E}$.

\section{NON-POINT TRANSFORMATIONS IN HAMILTON'S VARIATIONAL PRINCIPLE}

We assume now that the second-order equations (3.2) are the Euler-Lagrange equations (2.2) for Hamilton's variational principle

$$
\delta I \equiv \delta \int_{t_{1}}^{t_{2}} \mathcal{L}(q, \dot{q}, t) d t=0
$$


with the conditions

$$
\delta q_{i}\left(t_{1}\right)=\delta q_{i}\left(t_{2}\right)=0, \quad i=1, \ldots, r
$$

Using in (4.1) relations (2.13a) and (3.5) we obtain the equality

$$
\delta \int_{t_{1}}^{t_{2}} \tilde{L}(Q, \dot{Q}, \ddot{Q}, t) d t=0
$$

where

$$
\begin{aligned}
\tilde{L}(Q, \dot{Q}, \ddot{Q}, t) & =\mathcal{L}(q, \dot{q}, t) \\
& =\mathcal{L}(\varphi(Q, \dot{Q}, t), \zeta(Q, \dot{Q}, \ddot{Q}, t))
\end{aligned}
$$

Inasmuch as

$$
\delta q_{i}=\frac{\partial \varphi_{i}}{\partial Q_{j}} \delta Q_{j}+\frac{\partial \varphi_{i}}{\partial \dot{Q}_{j}} \delta \dot{Q}_{j}
$$

the sufficient conditions for satisfying (4.2) are:

$$
\delta Q_{j}\left(t_{1}\right)=\delta Q_{j}\left(t_{2}\right)=0, \quad \delta \dot{Q}_{j}\left(t_{1}\right)=\delta \dot{Q}_{j}\left(t_{2}\right)=0
$$

If one requires that conditions (4.2) are preserved for arbitrary functions $\varphi_{i}$, equalities (3.5) are also necessary conditions.

Transformations (2.13a) and (3.5) should be applied in the action integral (4.1) not only to the extremals of the functional $I$ (i.e. to the solutions of the secondorder equations (2.2)), but to arbitrary functions $q_{i}(t) \in$ $C^{1}\left[t_{1}, t_{2}\right]$. It is the reason why the derivatives $\ddot{Q}_{k}$ cannot be eliminated in (4.3). Therefore we come to the variational principle with higher derivatives, first investigated by M. Ostrogradsky. Corresponding equations of extremals, i.e. the Euler-Ostrogradsky equations, are as follows:

$$
\Lambda^{j} \tilde{L} \equiv \frac{d^{2}}{d t^{2}} \frac{\partial \tilde{L}}{\partial \ddot{Q}_{j}}-\frac{d}{d t} \frac{\partial \tilde{L}}{\partial \dot{Q}_{j}}+\frac{\partial \tilde{L}}{\partial Q_{j}}=0
$$

In general they are fourth-order equations.

The following theorem holds here:

Theorem 1 If functions $q_{i}(t)$ are extremals for the variational principle (4.1), (4.2), then functions $Q_{j}(t)$, which are connected with $q_{i}(t)$ by formulae (2.13a) and (3.5), are extremals for the variational principle (4.3), (4.6).

The proof (see Appendix 1) consists in establishing the equality

$$
\Lambda^{j} \tilde{L}=\frac{d}{d t}\left\{\frac{\partial q_{i}}{\partial \dot{Q}_{j}}\left(\frac{d}{d t} \frac{\partial \mathcal{L}}{\partial \dot{q}_{i}}-\frac{\partial \mathcal{L}}{\partial q_{i}}\right)\right\}
$$

$$
-\frac{\partial q_{i}}{\partial Q_{j}}\left(\frac{d}{d t} \frac{\partial \mathcal{L}}{\partial \dot{q}_{i}}-\frac{\partial \mathcal{L}}{\partial q_{i}}\right)
$$

from which the above theorem follows directly.

Remark 3. The applications of transformations (2.13a) and (3.5) to the Euler-Lagrange equations (2.2) without elimination of third derivatives $\dddot{q}_{i}$ by the method of the Section III leads to the third-order equations (3.6) for functions $Q_{j}(t)$. At the same time the use of these transformations in the variational principle (4.1), (4.2) results in fourth-order equations (4.7) for extremals. Inasmuch as these two sets of differential equations are consequences of the same equations (2.2) for $q_{i}(t)$ and the same transformations (2.13a) and (3.5), we conclude that they should have a common set $\mathfrak{M}_{(3)}$ of solutions, which is a subset of the set $\mathfrak{M}_{(4)}$ containing all solutions of the equations (4.7): $\mathfrak{M}_{(3)} \subset \mathfrak{M}_{(4)}$. In other words, the application of non-point transformations in the variational principle leads to wider class of functions $Q_{j}(t)$ associated with initial functions $q_{i}(t)$ in comparison with transformation of the Euler-Lagrange equations.

In the example considered above, the Lagrangian

$$
\mathcal{L}=\frac{\dot{x}^{2}}{2}-\frac{x^{2}}{2}
$$

corresponds to equation (3.13). It follows from (3.16a) that the transformed Lagrangian and the corresponding Euler-Ostrogradsky equation have the form

$$
\begin{gathered}
\tilde{L}=\frac{1}{2} \ddot{X}^{2}-\frac{1}{2} X^{2}+\frac{d}{d t}\left(\frac{\dot{X}^{2}-X^{2}}{2}\right), \\
\stackrel{(4)}{X}-X=0 .
\end{gathered}
$$

Every solution of (3.18) or (3.19) satisfies also equation (4.11). Taking into account that because of the identity

$$
\Lambda^{j} \frac{d f(Q, \dot{Q}, t)}{d t}=0
$$

(where $f$ is an arbitrary function) the addition of the total time-derivative to Lagrangian does not change the equations (4.7), we can drop the last term in (4.10).

\section{CONNECTION BETWEEN STANDARD LAGRANGIANS ASSOCIATED WITH NON-POINT TRANSFORMATIONS}

We suppose now (just as in Section IV) that equations (3.1) or (3.2) are Euler-Lagrange equations (2.2) for the variational principle with the standard, in classical mechanics, Lagrangian $\mathcal{L}(q, \dot{q}, t)$. Let, further, the 
connection between new and old variables be expressed by non-point transformations (2.12) or (2.13) which are constructed on the basis of $(2.12 \mathrm{a})$ or $(2.13 \mathrm{a})$, respectively, with use of equations (2.2). Then a question arises: how can one find the standard Lagrangian $L(Q, \dot{Q}, t)$ for which the Euler-Lagrange equations (2.3) coincide with new second-order equations (3.7) or (3.8)?

As it was noted in Section IV, function $L$ to be found cannot be obtained by means of direct elimination of accelerations from Lagrangian $\tilde{L}$ (4.4) with the use of equations (2.3) or (3.7) [12]. The reason resides in the fact that these equations themselves are consequences of variational principle

$$
\delta I \equiv \delta \int_{t_{1}}^{t_{2}} L(Q, \dot{Q}, t) d t=0
$$

in which the action integral $I$ is defined not only on its extremals but on arbitrary functions $Q_{k}(t) \in C^{1}\left[t_{1}, t_{2}\right]$. Nevertheless the following theorem holds:

Theorem 2 Let the non-point transformations (2.12) and (2.13) be given on the set of functions $q_{i}(t)$ which are, according to (4.1), extremals of the functional I; let, furthermore, function $F=F(q, Q, t)$ be an arbitrary solution of the set of the partial differential equations

$$
\frac{\partial F(q, Q, t)}{\partial q_{i}}=\left.\frac{\partial \mathcal{L}}{\partial \dot{q}_{i}}\right|_{(q, Q, t)},
$$

where in right-hand side the partial differentiation is followed by elimination of all variables $\dot{q}_{i}$ in terms of $(q, Q, t)$ using equations (2.12a): $\dot{q}_{i}=\mathcal{X}(q, Q, t)$. Then functions $Q_{j}(t)$ form the set of solutions of EulerLagrange equations for the Lagrangian

$$
\begin{aligned}
L(Q, \dot{Q}, t) & =\mathcal{L}(\varphi(Q, \dot{Q}, t), \psi(Q, \dot{Q}, t), t) \\
& -\left.\frac{d F(q, Q, t)}{d t}\right|_{(Q, \dot{Q}, t)},
\end{aligned}
$$

where in right-hand side the variables $q_{i}$ and $\dot{q}_{i}$ are $e x$ pressed in terms of $(Q, \dot{Q}, t)$ on the basis of the equalities (2.13).

The proof of Theorem 2 is based on the Lemma:

Lemma 1 The function L, defined by formula (5.3), satisfies the equality

$$
\frac{\partial L}{\partial \dot{Q}_{j}}=-\left.\frac{\partial F}{\partial Q_{j}}\right|_{(Q, \dot{Q}, t)}
$$

Here relations (2.13a) should be used after differentiating. The proof of the Lemma is given in Appendix 2.
To prove Theorem 2 (see Appendix 3) it is sufficient to show that the action of the Euler-Lagrange operator on the function $L(5.3)$ gives zero if the functions $q_{i}(t)$ satisfy the Euler-Lagrange equations (2.2).

As an example, the transformed Lagrangian $L$ will be constructed which corresponds to initial Lagrangian $\mathcal{L}(4.9)$ and to transformations (3.16a) and (3.20). The equation (5.2) is as follows:

$$
\frac{\partial F(x, X, t)}{\partial x}=x-2 X+2 C e^{-t}
$$

its general solution has the form

$$
F(x, X, t)=\frac{1}{2} x^{2}-2 X x+2 C e^{-t} x+F_{0}(X, t),
$$

where $F_{0}(X, t)$ is an arbitrary function (the general solution of corresponding homogeneous equation). The derivative $d F / d t$ can be expressed as follows:

$$
\begin{aligned}
& \left.\frac{d F}{d t}\right|_{(X, \dot{X}, t)}=-\dot{X}^{2}+X^{2}-4 C e^{-t} X \\
& -\frac{d}{d t}\left[2 X^{2}-2 C e^{-t} X+2 C^{2} e^{-2 t}-F_{0}(X, t)\right] .
\end{aligned}
$$

Using formula (5.3) and dropping the total derivative in (5.7) we obtain:

$$
L=\dot{X}^{2}-X^{2}+4 C e^{-t} X .
$$

The corresponding Euler-Lagrange equation is identical to $(3.19)$.

Remark 4. After the elimination of variables $(q, \dot{q})$ in formula $(5.3)$ by means of equalities (2.13) the last term of this formula is no more a total time-derivative and, therefore, it is essential for defining of Lagrangian $L(Q, \dot{Q}, t)$. The general solution of the set of nonhomogeneous differential equations (5.2) contains an arbitrary function $F_{0}(Q, t)$ as an additional term, for which the transformation is irrelevant. Thus, the expression $d F_{0} / d t$ in (5.3) preserves the sense of total time-derivative and has no influence on the form of Euler-Lagrange equations. It should be also noted that the ambiguity of $F$ and $L$ caused by the term $F_{0}$ can be removed by the natural requirement that the inverse transition $L(Q, Q, t) \rightarrow$ $\mathcal{L}(q, \dot{q}, t)$ results in the initial expression for $\mathcal{L}$. Of course, the derivative

$$
\frac{d f(Q, q, t)}{d t}=-\frac{d F(q, Q, t)}{d t}
$$

will be present in the corresponding formula (5.3) for $\mathcal{L}$ and, therefore, $f(Q, q, t)=-F(q, Q, t)$ (up to an arbitrary constant). The latter equality allows to fix unambiguously the function $F_{0}(Q, t)$ as well as its counterpart $f_{0}(q, t)$. Specifically, in our example we find 


$$
f(X, x, t)=2 x X-X^{2}+f_{0}(x, t) .
$$

Here the formula of the type (5.2) and equalities (5.8) and (3.14) have been used. Comparing (5.10) and (5.6) we obtain

$$
F_{0}(X, t)=X^{2}, \quad f_{0}(x, t)=\frac{1}{2} x^{2}-2 C e^{-t} x
$$

and, finally,

$$
F(X, x, t)=\frac{1}{2} x^{2}-2 x X+X^{2}+2 C e^{-t} x .
$$

Remark 5. Theorem 2, which was formulated without reference to the Hamiltonian formalism, can be considered as a reflection of well known relations in the theory of canonical transformations. Indeed, it follows from definition of canonical momenta

$$
p_{i}=\frac{\partial \mathcal{L}}{\partial \dot{q}_{i}}, \quad P_{j}=\frac{\partial L}{\partial \dot{Q}_{j}}
$$

that equalities (5.2) and (5.4) can be rewritten as follows

$$
p_{i}=\frac{\partial F}{\partial q_{i}}, \quad P_{j}=-\frac{\partial F}{\partial Q_{j}} .
$$

Here $F(q, Q, t)$ has the sense of the generating function of the canonical transformation $(q, p, t) \mapsto(Q, P, t)$, for which the well known conditions take place:

$$
\begin{gathered}
p_{i} \dot{q}^{i}-P_{i} \dot{Q}^{i}=\frac{d F}{d t}-\frac{\partial F}{\partial t}, \\
H(Q, P, t)-\mathcal{H}(q, p, t)=\frac{\partial F}{\partial t} .
\end{gathered}
$$

Combining relations (5.14) and (5.15) with Legendre transformation

$$
\mathcal{L}=p_{i} \dot{q}^{i}-\mathcal{H}, \quad L=P_{j} \dot{Q}^{j}-H
$$

we obtain equality (5.3).

In our example canonical transformations corresponding to function (5.12) have the form:

$$
X=\frac{x-p}{2}+C e^{-t}, \quad P=p+x-2 C e^{-t} .
$$

\section{DISCUSSION}

The first question which should be discussed is the meaning of the function $F(q, Q, t)$, which has been introduced in Section $\mathrm{V}$.
We note first of all that the right-hand sides of Eqs. (5.2) are defined by Lagrangian $\mathcal{L}$ as well as by the functions $\mathcal{X}_{i}(q, Q, t)$ which can be found, in their turn, from non-point transformations (2.12). Taking into account that the latter transformations follow (when $\mathcal{L}$ is given) equalities (2.12a) or (2.13a), we conclude: relations $(2.12 \mathrm{a})$ or $(2.13 \mathrm{a})$ together with the initial Lagrangian define the structure of the set of equations (5.2) and, therefore, the function $F(q, Q, t)$ (up to an arbitrary additive term $\left.F_{0}(Q, t)\right)$.

On the other hand, if function $F(q, Q, t)$ and Lagrangian $\mathcal{L}(q, \dot{q}, t)$ are given we may consider (5.2) as a set of equations allowing to find the functions $Q_{i}=$ $\Phi_{i}(q, \dot{q}, t)$. Solving these equations and using the methods of Sec. III leads to the complete set of non-point transformations (2.12) and (2.13). Therefore, the function $F(q, Q, t)$ may be called generating function of these transformations. Nevertheless, this term should not be interpreted conditionally inasmuch as its meaning differs somewhat from that typical for generating function $F(q, Q, t)$ of canonical transformations. The function $F(q, Q, t)$ defines canonical transformation in the $2 r$-dimensional phase space of an arbitrary system completely, whereas finding transformations (2.12) and (2.13) requires additionally the knowledge of the Lagrangian $\mathcal{L}(q, \dot{q}, t)$. Thus, some totality of transformations of the type (2.12) or (2.13) corresponds to the given function $F(q, Q, t)$. It follows from Eqs. (5.2) that the difference between such transformations arises in cases when Lagrangians of two systems with the same number of degrees of freedom have a different dependence on velocities. Inasmuch as the structures of free-particle Lagrangians coincide for all systems with a fixed number of degrees of freedom, the above mentioned difference has place when interaction depends on velocities and the dependence differs for two interaction Lagrangians. Such a situation occurs, especially, in quasirelativistic mechanics of particle systems [3]. For instance, post-Newtonian Lagrangians for electromagnetic and gravitational interactions (i.e. the Darwin's and Fichtenholtz's Lagrangians) have essentially different structure. In such cases different non-point transformations in Lagrangian and Newtonian formalisms correspond to the same canonical transformations in Hamiltonian formalism.

The second point worth noting is the connection between the standard Lagrangians $\mathcal{L}(q, \dot{q}, t)$ and $L(Q, \dot{Q}, t)$ when the variables $(q, \dot{q})$ and $(Q, \dot{Q})$ are connected by non-point transformations (see Theorem 2). It follows from our results that the assertion of [15] about the lack of such a connection is not accurate. This connection does not exist, in fact, in the action integral $I$ and in variational principle (5.1) because transformations (2.12) and (2.13) hold only for extremals of the functional. Nevertheless, in accordance with equality (5.3), a new standard Lagrangian $L$ can be constructed, and the corresponding new equations of motion, as well as conservation laws in terms of new variables, can be formulated in the framework of the Lagrangian formalism. The Lagrangian $L(Q, \dot{Q}, t)$ is not the result of the elimination of the second derivatives $\ddot{Q}_{j}$ in the Ostrogradsky 
Lagrangian $\tilde{L}(Q, \dot{Q}, \ddot{Q}, t)$ (see Section IV) which is obtained by means of mapping $(q, \dot{q}) \mapsto(Q, \dot{Q}, \ddot{Q})$ without using the initial Euler-Lagrange equations. These final remarks indicate a subtle distinction between point and non-point transformations in the Lagrangian formalism.

I should like to thank Yu. B. Kluchkovskyi, R. Ya. Matsyuk, and V. I. Tretyak for many interesting and useful discussions.

\section{Appendix 1}

The proof of equality (4.8) consists in the transformation of the expression

$$
\Lambda^{j} \tilde{L} \equiv \frac{d^{2}}{d t^{2}} \frac{\partial \tilde{L}}{\partial \ddot{Q}_{j}}-\frac{d}{d t} \frac{\partial \tilde{L}}{\partial \dot{Q}_{j}}+\frac{\partial \tilde{L}}{\partial Q_{j}}
$$

using (2.13a) and (4.4). We remember that the use of $(2.13 \mathrm{~b})$ would be incorrect in the variational principle (4.1) or (4.3) and in the Eqs. (4.7) before solving the latter to find extremals.
Equalities (2.13a) and (3.5) imply the formula

$$
\dot{q}_{i}=\frac{\partial q_{i}}{\partial Q_{k}} \dot{Q}_{k}+\frac{\partial q_{i}}{\partial \dot{Q}_{k}} \ddot{Q}_{k}+\frac{\partial q_{i}}{\partial t}
$$

from which it follows that

$$
\frac{\partial \dot{q}_{i}}{\partial \ddot{Q}_{j}}=\frac{\partial q_{i}}{\partial \dot{Q}_{j}}
$$

$$
\frac{\partial \dot{q}_{i}}{\partial \dot{Q}_{j}}=\frac{d}{d t} \frac{\partial q_{i}}{\partial \dot{Q}_{j}}+\frac{\partial q_{i}}{\partial Q_{j}}
$$

and

$$
\frac{\partial \dot{q}_{i}}{\partial Q_{j}}=\frac{d}{d t} \frac{\partial q_{i}}{\partial Q_{j}}
$$

Using relations (A1.3)-(A1.5) we obtain the following:

$$
\begin{gathered}
\frac{d^{2}}{d t^{2}} \frac{\partial \tilde{L}}{\partial \ddot{Q}_{j}}=\frac{d^{2}}{d t^{2}}\left(\frac{\partial \mathcal{L}}{\partial \dot{q}_{i}} \frac{\partial \dot{q}_{i}}{\partial \ddot{Q}_{j}}\right)=\frac{d^{2}}{d t^{2}}\left(\frac{\partial \mathcal{L}}{\partial \dot{q}_{i}} \frac{\partial q_{i}}{\partial \dot{Q}_{j}}\right)=\frac{d}{d t}\left[\frac{\partial q_{i}}{\partial \dot{Q}_{j}} \frac{d}{d t}\left(\frac{\partial \mathcal{L}}{\partial \dot{q}_{i}}\right)+\frac{\partial \mathcal{L}}{\partial \dot{q}_{i}} \frac{d}{d t}\left(\frac{\partial q_{i}}{\partial \dot{Q}_{j}}\right)\right] \\
\frac{d}{d t} \frac{\partial \tilde{L}}{\partial \dot{Q}_{j}}=\frac{d}{d t}\left(\frac{\partial \mathcal{L}}{\partial q_{i}} \frac{\partial q_{i}}{\partial \dot{Q}_{j}}+\frac{\partial \mathcal{L}}{\partial \dot{q}_{i}} \frac{\partial \dot{q}_{i}}{\partial \dot{Q}_{j}}\right)=\frac{d}{d t}\left[\frac{\partial \mathcal{L}}{\partial q_{i}} \frac{\partial q_{i}}{\partial \dot{Q}_{j}}+\frac{\partial \mathcal{L}}{\partial \dot{q}_{i}}\left(\frac{d}{d t} \frac{\partial q_{i}}{\partial \dot{Q}_{j}}+\frac{\partial q_{i}}{\partial \dot{Q}_{j}}\right)\right] \\
\frac{\partial \tilde{L}}{\partial Q_{j}}=\frac{\partial \mathcal{L}}{\partial q_{i}} \frac{\partial q_{i}}{\partial Q_{j}}+\frac{\partial \mathcal{L}}{\partial \dot{q}_{i}} \frac{\partial \dot{q}_{i}}{\partial Q_{j}}=\frac{\partial \mathcal{L}}{\partial q_{i}} \frac{\partial q_{i}}{\partial Q_{j}}+\frac{\partial \mathcal{L}}{\partial \dot{q}_{i}} \frac{d}{d t} \frac{\partial q_{i}}{\partial Q_{j}}=\frac{d}{d t}\left(\frac{\partial \mathcal{L}}{\partial \dot{q}_{i}} \frac{\partial q_{i}}{\partial Q_{j}}\right)+\frac{\partial q_{i}}{\partial Q_{j}}\left(\frac{\partial \mathcal{L}}{\partial q_{i}}-\frac{d}{d t} \frac{\partial \mathcal{L}}{\partial \dot{q}_{i}}\right)
\end{gathered}
$$

Inserting (A1.6)-(A1.8) into (A1.1) we obtain (4.8).

\section{Appendix 2}

We prove the Lemma expressed by the relation

$$
\frac{\partial L}{\partial \dot{Q}_{j}}=-\left.\frac{\partial F}{\partial Q_{j}}\right|_{(Q, \dot{Q}, t)}
$$

where $L$ is presented by equality (5.3) and function F satisfies equation (5.2). To do that it is sufficient to carry out the formal transformations in the left-hand side of the relation (A2.1) using (5.3), (2.2), and (5.2):

$$
\frac{\partial L}{\partial \dot{Q}_{j}}=\frac{\partial \mathcal{L}}{\partial q_{i}} \frac{\partial q_{i}}{\partial \dot{Q}_{j}}+\frac{\partial \mathcal{L}}{\partial \dot{q}_{i}} \frac{\partial \dot{q}_{i}}{\partial \dot{Q}_{j}}-\frac{\partial}{\partial \dot{Q}_{j}}\left\{\left.\frac{d F}{d t}\right|_{(Q, \dot{Q}, t)}\right\}
$$




$$
\begin{gathered}
\frac{\partial \mathcal{L}}{\partial q_{i}}=\frac{d}{d t} \frac{\partial \mathcal{L}}{\partial \dot{q}_{i}}=\frac{d}{d t} \frac{\partial F}{\partial q_{i}}=\frac{\partial^{2} F}{\partial q_{i} \partial q_{k}} \dot{q}_{k}+\frac{\partial^{2} F}{\partial q_{i} \partial Q_{k}} \dot{Q}_{k}+\frac{\partial^{2} F}{\partial t \partial q_{i}} \\
\frac{\partial}{\partial \dot{Q}_{j}}\left\{\left.\frac{d F}{d t}\right|_{(Q, \dot{Q}, t)}\right\}=\frac{\partial}{\partial \dot{Q}_{j}}\left\{\left.\left(\frac{\partial F}{\partial q_{k}} \dot{q}_{k}+\frac{\partial F}{\partial Q_{k}} \dot{Q}_{k}+\frac{\partial F}{\partial t}\right)\right|_{(Q, \dot{Q}, t)}\right\} \\
=\frac{\partial^{2} F}{\partial q_{i} \partial q_{k}} \frac{\partial q_{i}}{\partial \dot{Q}_{j}} \dot{q}_{k}+\frac{\partial F}{\partial q_{k}} \frac{\partial \dot{q}_{k}}{\partial \dot{Q}_{j}}+\frac{\partial^{2} F}{\partial q_{i} \partial Q_{k}} \frac{\partial q_{i}}{\partial \dot{Q}_{j}} \dot{Q}_{k}+\frac{\partial F}{\partial Q_{j}}+\frac{\partial^{2} F}{\partial t \partial q_{i}} \frac{\partial q_{i}}{\partial \dot{Q}_{j}}
\end{gathered}
$$

Inserting (A2.3), (5.2), and (A2.4) into (A2.2) we see that the relation (A2.1) is true.

\section{Appendix 3}

The proof of Theorem 2 is based on the use of relations (5.4) and (5.3) which allow to present the left-hand side of Euler-Lagrange equations (2.3) as follows:

$$
\frac{d}{d t} \frac{\partial L}{\partial \dot{Q}_{j}}-\frac{\partial L}{\partial Q_{j}}=-\frac{d}{d t} \frac{\partial F}{\partial Q_{j}}-\frac{\partial}{\partial Q_{j}}\left\{\left.\mathcal{L}\right|_{(Q, \dot{Q}, t)}\right\}+\frac{\partial}{\partial Q_{j}}\left\{\left.\frac{d F}{d t}\right|_{(Q, \dot{Q}, t)}\right\}
$$

Taking into account Eqs. (2.2) we find

$$
\left.\frac{\partial}{\partial Q_{j}} \mathcal{L}\right|_{(Q, \dot{Q}, t)}=\frac{\partial \mathcal{L}}{\partial q_{i}} \frac{\partial q_{i}}{\partial Q_{j}}+\frac{\partial \mathcal{L}}{\partial \dot{q}_{i}} \frac{\partial \dot{q}_{i}}{\partial Q_{j}}=\left(\frac{d}{d t} \frac{\partial F}{\partial q_{i}}\right) \frac{\partial q_{i}}{\partial Q_{j}}+\frac{\partial F}{\partial q_{i}} \frac{\partial \dot{q}_{i}}{\partial Q_{j}}
$$

Also, we have

$$
\left.\frac{\partial}{\partial Q_{j}} \frac{d F}{d t}\right|_{(Q, \dot{Q}, t)}=\frac{\partial}{\partial Q_{j}}\left\{\left.\left(\frac{\partial F}{\partial q_{k}} \dot{q}_{k}+\frac{\partial F}{\partial Q_{k}} \dot{Q}_{k}+\frac{\partial F}{\partial t}\right)\right|_{(Q, \dot{Q}, t)}\right\} .
$$

Inserting (A3.2) and (A3.3) into (A3.1) and performing necessary calculations one can see that the right-hand side of equality (A3.1) becomes into zero. This completely proves Theorem 2.

[1] D. G. Currie, J. Math. Phys. 4, 1470 (1963).

[2] D. G. Currie, T. F. Jordan, E. C. G. Sudarshan, Rev. Mod. Phys. 35, 350 (1963).

[3] R. P. Gaida, Sov. J. Part. Nucl. 13, 179 (1982).

[4] R. P. Gaida, Yu. B. Kluchkovsky, V. I. Tretyak, in Constraint's Theory and Relativistic Dynamics, edited by G. Longhi, L. Lusanna (World Scientific Publ., Singapore, 1987), p. 210.

[5] R. P. Gaida, V. I. Tretyak, Yu. G. Yaremko, Ukr. Fiz. Zh. 36, 1807 (1991).

[6] R. P. Gaida, Dokl. Acad. Nauk Ukr. SSR A 1, 32 (1982).

[7] Y. Takahashi, Fortschr. Phys. 37, 443 (1989).

[8] Yu. I. Manin, in Itogi nauki i tiekhniki. Sovriemiennyie probliemy matiematiki, tom 11 (Modern Problems in Mathematics, vol. 11) (VINITI, Moscow, 1978), p. 152.

[9] F. Klein, Vorlesungen über höhere Geometrie (Springer, Berlin, 1926).

[10] S. A. Vladimirov, Gruppy simmietrii differientsial'nykh uravnienii $i$ rieliativistskiie polia (Symmetry Groups of Differential Equations and Relativistic Fields) (Atomizdat, Moscow, 1979).

[11] R. L. Anderson, N. H. Ibragimov, Lie-Bäcklund Transformations in Applications (SIAM, Philadelphia, 1979).

[12] Incorrectness of such an operation has not been considered in the paper [13] (see also [14]) where accelerations were eliminated in $c^{-4}$-terms of the function $\tilde{L}$ using non-relativistic equations of motion; the function obtained in this way no longer has the meaning of a Lagrangian.

[13] V. N. Golubenkov, Ya. A. Smorodinskiy, Zh. Eksp. Teor. Fiz. 31, 330 (1956).

[14] B. M. Barker, R. F. O'Connel, Ann. Phys. (N.Y.) 129, 358 (1980).

[15] J. W. Leech, Classical Mechanics (Wiley, New York, 1958). 


\title{
НЕТОЧКОВІ ПЕРЕТВОРЕННЯ У КЛ АСИЧНІЙ МЕХАНІЦІ
}

\author{
Р. П. Гайда \\ Інститут фізики конденсованих систем НАН Украӥни, \\ вул. Свенціцвкого, 1, Львів, 290011, Украӥна
}

\begin{abstract}
Дослід жено проблему застосовности неточкових перетворень у лагран жевому й ньютоновому формалізмах класичної механіки. Ці перетворення відповідають неточковим канонічним перетворенням і нале жать до класу перетворень, дотичних до заданої системи диференційних рівнянь другого порядку. Розглянуто методи побу дови таких перетворень. Доведено дві теореми про неточкові перетворення у варіяційному принципі Гамільтона і про співвідношення між перетвореним і вихідним лагранжіянами. Обговорено зв'язок отриманих результатів з канонічними перетвореннями гамільтонового формалізму. Загальні результати проілюстровано простим прикладом.
\end{abstract}

